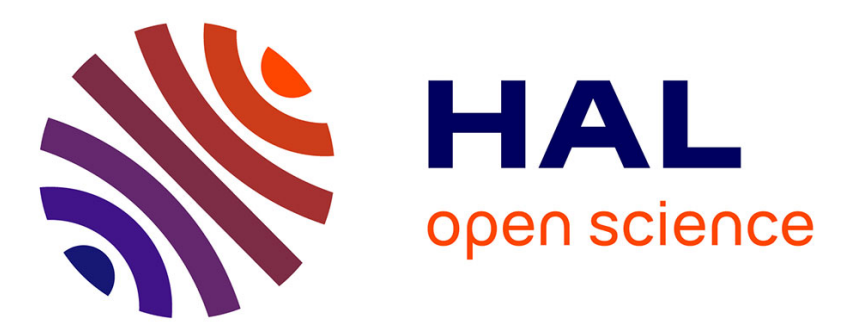

\title{
A finite volume scheme for hybrid turbulent two-phase flow models
}

\author{
Jean-Marc Hérard, Jean-Pierre Minier, Sergio Chibarro
}

\section{To cite this version:}

Jean-Marc Hérard, Jean-Pierre Minier, Sergio Chibarro. A finite volume scheme for hybrid turbulent two-phase flow models. 18th AIAA Computational Fluid Dynamics Conference, Jun 2007, New Orleans, United States. pp.2112-2122, 10.2514/6.2007-4587 . hal-01582668

\section{HAL Id: hal-01582668 \\ https://hal.science/hal-01582668}

Submitted on 5 Apr 2018

HAL is a multi-disciplinary open access archive for the deposit and dissemination of scientific research documents, whether they are published or not. The documents may come from teaching and research institutions in France or abroad, or from public or private research centers.
L'archive ouverte pluridisciplinaire HAL, est destinée au dépôt et à la diffusion de documents scientifiques de niveau recherche, publiés ou non, émanant des établissements d'enseignement et de recherche français ou étrangers, des laboratoires publics ou privés. 


\title{
A Finite Volume Scheme For Hybrid Turbulent Two-Phase Flow Models
}

\author{
Jean-Marc Hérard, Jean-Pierre Minier * \\ EDF, RED, 78400, Chatou, France
}

\author{
Sergio Chibbaro ${ }^{\dagger}$ \\ Universita di Roma, 00133, Roma, Italy
}

\begin{abstract}
In this paper, we present a new hybrid method for the computation of dispersed twophase flows. As with the classical hybrid method, an Eulerian (or mean-field) and a Lagrangian (or PDF) points of view are coupled but, in the present approach, the hybrid Euler/Lagrange description is extended to the simulation of the particle phase. In particular, the particle volumetric fraction and the particle mean velocity are obtained as solutions of partial differential equations on a mesh where a part of the information is provided by the Lagrangian description as a source term in the evolution equations. The second objective of the paper is to propose a novel numerical technique which relies on the hyperbolic nature of the underlying equations and makes use of relaxation techniques. The numerical ideas are explained and first computational results illustrate the feasability of the new hybrid method.
\end{abstract}

\section{Introduction}

Polydispersed turbulent two-phase flows are turbulent flows in which particles of different sizes are embedded. Accurate models and numerical simulations are useful for many industrial processes, particularly combustion devices, and also for environmental purposes (for example, particle dispersion in the atmosphere) and for new green energy-producing techniques. Modelling and simulating turbulent two-phase flows require to couple different physical descriptions for the fluid and the particle phases. In present models, the fluid phase is always described by a macroscopic Eulerian approach, while the particle phase is either described by an Eulerian approach (modelling constitutive relations for averaged properties) or by a Lagrangian description (modelling the instantaneous behaviour of individual elements). The Lagrangian approach, which provides information on the whole pdf, is best suited to address complex physical terms while the Eulerian, which only provides information on first and second moments, is simpler and numerically more efficient. Thus, most numerical approaches are based on a separate choice : either Eulerian or Lagrangian. A new possibility is to develop a multi-scale, or hybrid, method that would combine and use the two above-mentioned descriptions at the same time.

Similar ideas of hybrid methods can also be applied for single phase turbulent flows. In that case, it is tempting to compute local instantaneous values of the Reynolds stress components using the evaluation of particle velocity fluctuations provided by the Lagrangian solver. One difficulty -among others- in that case consists in solving the Reynolds stress averaged equations for the mean density, mean momentum and mean total energy while prescribing the Reynolds stress tensor computed by the Lagrangian code. This has already been examined by S.B. Pope and co-workers for instance $\left(\mathrm{see}^{1}\right)$. However, this approach requires a very accurate and stable implementation of so-called source terms (that is, the divergence of the Reynolds stress tensor in practice in that particular case). It was suggested $i^{2}$ that an efficient way to meet that requirement consists in mimicking the computation of a "father" system, in order to stabilize numerical approximations in a meaningful manner. Otherwise, the numerical pollution might destroy the whole effort of this new approach.

The work reported herein starts from a similar remark. The main idea is to retain a Lagrangian description of the dispersed phase while calculating the first two statistical moments, namely the volumetric fraction

\footnotetext{
*Département MFEE, 6 quai Watier

${ }^{\dagger}$ Postdoctoral position, Tor Vergata
} 
and the particle mean velocity, through an Eulerian formulation. An important improvement of the method is to get rid of the bias error by computing the first two statistical moments as the solution of a set of partial differential equations. Therefore, one needs to compute a coupled system including mass and momentum equations for particles, keeping in mind the fact that a kinetic tensor is provided by the Lagrangian code. This will be presented in the following section. In the present formulation, the key point is to introduce a father model in order to compute Eulerian variables. Then, as put forward in the next three sections, we adress successively the one dimensional and the two dimensional frameworks, and the numerical schemes, which rely on the combined use of upwinding techniques and relaxation algorithms, are detailed. The computational results described herein aim at verifying that the pollution due to the numerical schemes is far less than the "true" pollution caused by noise.

\section{Presentation of the hybrid method}

The hybrid method requires computing accurate approximations of the two main unknowns $\alpha_{p}(t, \mathbf{x})$ and $\left\langle\mathbf{U}_{p}\right\rangle(t, \mathbf{x})$.

The mean equations for $\alpha_{p}^{E}(t, \mathbf{x})$ and $\left\langle\mathbf{U}_{p}^{E}\right\rangle(t, \mathbf{x})$ corresponding to our hybrid model are:

$$
\begin{gathered}
\frac{\partial \alpha_{p}^{E} \rho_{p}}{\partial t}+\nabla \cdot\left(\alpha_{p}^{E} \rho_{p}\left\langle\mathbf{U}_{p}\right\rangle^{E}\right)=0 \\
\alpha_{p}^{E} \rho_{p} \frac{\partial\left\langle\mathbf{U}_{p}\right\rangle^{E}}{\partial t}+\alpha_{p}^{E} \rho_{p}\left\langle\mathbf{U}_{p}\right\rangle^{E} \cdot \nabla\left\langle\mathbf{U}_{p}\right\rangle^{E}+\nabla \cdot \alpha_{p}^{E} \rho_{p}\left\langle\mathbf{u}_{p}^{\prime} \mathbf{u}_{p}^{\prime}\right\rangle^{L}=\alpha_{p}^{E} \rho_{p} \mathbf{g}+\left\langle\frac{\alpha_{p}^{E} \rho_{p} \mathbf{U}_{\mathbf{r}}}{\tau_{p}}\right\rangle
\end{gathered}
$$

Variables provided by the Lagrangian solver to the Eulerian one are indicated by a script " $L$ ". Initial conditions for both $\alpha_{p}^{E}(t, \mathbf{x})$ and $\left\langle\mathbf{U}_{p}\right\rangle^{E}(t, \mathbf{x})$ must be provided. Moreover, the cell values of the kinetic "Reynolds stress" tensor $\left\langle\mathbf{u}_{p}^{\prime} \mathbf{u}_{p}^{\prime}\right\rangle^{L}$ computed by means of the Lagrangian code are assumed to be given as a distribution of discrete local components, which of course will comply with the realisability concept (see $\left.{ }^{3}\right)$, since :

$$
0 \leq \mathbf{n}^{\mathbf{t}}\left\langle\mathbf{u}_{p}^{\prime} \mathbf{u}_{p}^{\prime}\right\rangle^{L} \mathbf{n}
$$

Afterwards, the mean velocity $\left\langle U_{p}\right\rangle^{E}$ is plugged into the Lagrangian stochastic method. The stochastic equations which need to be solved are recalled in. ${ }^{4}$ The advantage of the whole formulation is that the Eulerian moments are by definition not-biased and free of statistical noise. The issue of consistency between the Eulerian and Lagrangian predictions:

$$
\begin{aligned}
\alpha_{p}^{E} & =\alpha_{p}^{L} \\
\left\langle U_{p}\right\rangle^{E} & =\left\langle U_{p}\right\rangle^{L} .
\end{aligned}
$$

is discussed in. ${ }^{5-7}$

\section{Finite Volume method for the prediction of particle moments}

The kinetic tensor term provided by the Lagrangian solver is noisy, and may be seen as a highly discontinuous signal. Thus the computation of approximations of solutions of the set of equations (1)-(2) is rather difficult. In a pure Eulerian framework, one already knows that similar sets of equations require a specific treatment. Otherwise, instabilities may easily develop, especially in regions where the mean velocity field is weak compared with the amplitude (the trace) of the kinetic tensor. Euler type solvers may be used, omitting the fact that one needs to compute the divergence of the quantity $\alpha_{p}^{E} \rho_{p}\left\langle\mathbf{u}_{p}^{\prime} \mathbf{u}_{p}^{\prime}\right\rangle^{L}$. This simple approach has already been applied $\left(\mathrm{see}^{1}\right)$, but it suffers from severe drawbacks, as indicated in $^{8-10}$ for instance. Turning then to the mixed Eulerian / Lagrangian approach such as the one considered in this paper, the problem becomes even more tricky, since the kinetic tensor acts as an external input with discontinuous data, which inhibits using centered schemes to compute its divergence.

The approach that is proposed herein takes these facts into account, and relies on basic ideas which are similar to those discussed in. ${ }^{2}$ The main idea consists in constructing a father model, and then in using the 
hyperbolic nature of this father model in combination with an instantaneous relaxation method. In order to simplify the presentation, we get rid of sub and upper scripts, and consider the set of governing equations:

$$
\begin{aligned}
\frac{\partial \alpha}{\partial t} & +\nabla \cdot(\alpha\langle\mathbf{U}\rangle)=0 \\
\frac{\partial \alpha\langle\mathbf{U}\rangle}{\partial t} & +\nabla \cdot(\alpha\langle\mathbf{U}\rangle \otimes\langle\mathbf{U}\rangle)+\nabla \cdot\left(\alpha \mathbf{R}_{\text {Lag }}\right)=\mathbf{S} \\
\text { with } \mathbf{S} & =\alpha \mathbf{g}+\left\langle\frac{\alpha \mathbf{U}_{\mathbf{r}}}{\tau_{p}}\right\rangle ;
\end{aligned}
$$

The system can be re-written in a more compact form for the vector $\mathbf{W}=(\alpha, \mathbf{U})$

$$
\frac{\partial \mathbf{W}}{\partial t}+\nabla \cdot\left(\mathbf{F}^{1}(\mathbf{W})\right)+\nabla \cdot\left(\mathbf{F}^{2}\left(\mathbf{W}, \mathbf{R}_{\text {Lag }}\right)\right)=\mathcal{S}(\mathbf{W}, \mathbf{x}, t)
$$

with:

$$
\begin{cases}\mathbf{F}^{2}\left(\mathbf{W}, \mathbf{R}_{\text {Lag }}\right) & =\left(0, \alpha \mathbf{R}_{\text {Lag }}\right) \\ \mathcal{S} & =(0, \mathbf{S})\end{cases}
$$

The left hand side of the vector equation Eq. (9) has a pure advective nature and is under conservative form. Even if the source term $\mathbf{S}$ is put to zero, the system becomes numerically unstable in simple wall-boundary flows $^{8}$ if a centered scheme is used for the advective term $\mathbf{F}^{2}\left(\mathbf{W}, \mathbf{R}_{\text {Lag }}\right)$. In order to develop a stable numerical method, we decided to take advantage of recent computations which have been carried out within the framework of single-phase turbulent compressible flows $\left({ }^{2}\right)$.

We now introduce the father model as follows:

$$
\left\{\begin{array}{l}
\frac{\partial \alpha}{\partial t}+\nabla \cdot(\alpha\langle\mathbf{U}\rangle)=0 \\
\frac{\partial \alpha\langle\mathbf{U}\rangle}{\partial t}+\nabla \cdot(\alpha\langle\mathbf{U}\rangle \otimes\langle\mathbf{U}\rangle)+\nabla \cdot(\alpha \mathbf{R})=\mathbf{S} \\
\frac{\partial \alpha \mathbf{R}}{\partial t}+\nabla \cdot(\alpha\langle\mathbf{U}\rangle \mathbf{R})+\alpha \mathbf{R} \nabla\langle\mathbf{U}\rangle+\alpha \nabla^{\dagger}\langle\mathbf{U}\rangle \mathbf{R}=\mathbf{M}+\frac{\alpha}{\tau} \frac{\left(\left(\mathbf{R}_{\text {Lag }}-\mathbf{R}\right) R+R\left(\mathbf{R}_{\text {Lag }}-\mathbf{R}\right)\right)}{\operatorname{trace}(\mathbf{R})}
\end{array}\right.
$$

while noting $\alpha=\alpha_{p} \rho_{p}$. $\mathbf{M}$ is the model that must be consistent with the PDF model chosen here, whose expression is given in: ${ }^{11}$

$$
M_{i j}=-\frac{\partial}{\partial x_{k}}\left(\alpha\left\langle u_{i} u_{k} u_{k}\right\rangle\right)+\alpha\left[\left\langle\left(\frac{U_{s, i}-U_{p, i}}{\tau_{p}}\right) u_{j}\right\rangle+\left\langle\left(\frac{U_{s, j}-U_{p, j}}{\tau_{p}}\right) u_{i}\right\rangle\right] .
$$

The second term of the right hand side of the governing equation for the kinetic tensor is a relaxation term governed by its time scale $\tau$.

The algorithm consists in two steps :

- An evolution step

- A relaxation step

The evolution step requires the initialisation of both $\alpha$ and $\langle\mathbf{U}\rangle$, which is achieved with the Eulerian code. It needs to be fed with the reconstructed cell values of the kinetic tensor, using the Lagrangian code.

(1) Evolution: with given $\mathbf{z}^{n}=(\alpha,\langle\mathbf{U}\rangle, \mathbf{R})^{n}$, one computes approximations of solutions of the homogeneous system of equations (9):

$$
\left\{\begin{array}{l}
\frac{\partial \alpha}{\partial t}+\nabla \cdot(\alpha\langle\mathbf{U}\rangle)=0 \\
\frac{\partial \alpha\langle\mathbf{U}\rangle}{\partial t}+\nabla \cdot(\alpha\langle\mathbf{U}\rangle \otimes\langle\mathbf{U}\rangle)+\nabla \cdot(\alpha \mathbf{R})=0 \\
\frac{\partial \alpha \mathbf{R}}{\partial t}+\nabla \cdot(\alpha\langle\mathbf{U}\rangle \mathbf{R})+\alpha \mathbf{R} \nabla\langle\mathbf{U}\rangle+\alpha \nabla^{\dagger}\langle\mathbf{U}\rangle \mathbf{R}=0
\end{array}\right.
$$

This provides a first prediction $\mathbf{z}^{\star}$. 
(2) Relaxation: for given $\mathbf{z}^{\star}$, one computes successively approximations of the non homogeneous system:

$$
\left\{\begin{array}{c}
\frac{\partial \alpha}{\partial t}=0 \\
\frac{\partial \alpha\langle\mathbf{U}\rangle}{\partial t}=\mathbf{S} \\
\frac{\partial \alpha \mathbf{R}}{\partial t}=0 .
\end{array}\right.
$$

and then of:

$$
\left\{\begin{aligned}
\frac{\partial \alpha}{\partial t} & =0 \\
\frac{\partial \alpha\langle\mathbf{U}\rangle}{\partial t} & =0 \\
\frac{\partial \alpha \mathbf{R}}{\partial t} & =\mathbf{M}+\alpha\left(\left(\mathbf{R}_{\text {Lag }}-\mathbf{R}\right) R+R\left(\mathbf{R}_{\text {Lag }}-\mathbf{R}\right)\right) / \tau / \operatorname{trace}(\mathbf{R}) \quad \text { with } \tau \rightarrow 0
\end{aligned}\right.
$$

which eventually provides $\mathbf{z}^{n+1}$.

- The first step corrects the velocity field by accounting for drag and buoyancy effects. Both the void fraction and the kinetic tensor remain steady within this step.

- This second relaxation step is a ghost step for both the void fraction and the mean particle velocity. When $\tau$ is set to 0 , the kinetic tensor is relaxed to its equilibrium value provided by the Lagrangian code:

$$
\mathbf{R}^{n+1}=\mathbf{R}_{\text {Lag }}^{n+1} .
$$

\section{Main properties}

\section{A. The evolution step}

\section{Property 1}

In the one dimensional framework, the homogeneous system (13) is strictly hyperbolic, unless vacuum arises in the solution $(\alpha=0)$, or if the kinetic tensor is null. Noting $R=R_{11}$ and $c^{2}=3 R$, eigenvalues are:

$$
\begin{array}{r}
\lambda_{1}=U-c \\
\lambda_{2}=U \\
\lambda_{3}=U+c
\end{array}
$$

The 1 - and 3-fields are genuinely non linear and the 3 -field is linearly degenerated.

\section{Property 2}

We note $s=R /(\alpha)^{2}$. Then, in the one-dimensional framework, the Riemann problem associated with the homogeneous system (13) admits a unique solution with no vacuum occurence and realisable kinetic values provided that the initial data $\left(\mathbf{W}_{L}, R_{L}, \mathbf{W}_{R}, R_{R}\right)$ on each side of the initial discontinuity (located in $x=0$ ) comply with the condition:

$$
U_{R}-U_{L}<\int_{0}^{(\alpha R)_{L}}\left(\frac{1}{(\alpha c)\left(s_{L}, \alpha R\right)}\right) d(\alpha R)+\int_{0}^{(\alpha R)_{R}}\left(\frac{1}{(\alpha c)\left(s_{R}, \alpha R\right)}\right) d(\alpha R)
$$

and restricting to weak shock waves. If the condition (20) is violated, a vacuum of particles occurs in the solution.

\section{B. The relaxation step}

The relaxation step guarantees that: 
- The void fraction $\alpha$ remains positive.

- The kinetic tensor $\mathbf{R}$ remains realisable, even if one considers a physical relaxation step (when $\tau$ is non-zero) in (11). The proof is similar to the one given in. ${ }^{12}$

\section{Extension to a multi-dimensional framework}

If we turn now to a two-dimensional framework, similar results hold. The counterpart of the previous properties is slightly changed since new fields occur in the Riemann problem associated with the homogeneous part (11).Before going further on, we need to introduce some new notations. Given some unit normal vector $\mathbf{n}=\left(n_{x}, n_{y}\right)$, we set $\mathbf{n}^{T}=\left(-n_{y}, n_{x}\right), U_{n}=\mathbf{U} . \mathbf{n}, U_{T}=\mathbf{U} \cdot \mathbf{n}^{T}$ and also $R_{n n}=\mathbf{n}^{t} \mathbf{R} \mathbf{n}, R_{n \tau}=\mathbf{n}^{t} \mathbf{R} \mathbf{n}^{T}$ and $R_{\tau \tau}=\left(\mathbf{n}^{T}\right)^{t} \mathbf{R n}^{T}$.

Property 3

In a two-dimensional framework, the homogeneous system (13) is hyperbolic, unless vacuum arises in the solution $(\alpha=0)$, or if the kinetic tensor $\mathbf{R}$ is null. Noting $c_{1}^{2}=3 R_{n n}$ and $c_{2}^{2}=R_{n n}$, eigenvalues are:

$$
\begin{gathered}
\lambda_{1}=U_{n}-c_{1} \\
\lambda_{2}=U_{n}-c_{2} \\
\lambda_{3,4}=U_{n} \\
\lambda_{5}=U_{n}+c_{2} \\
\lambda_{6}=U_{n}+c_{1}
\end{gathered}
$$

The 1- and 6-fields are genuinely non linear and the 2,3,4,5-fields are linearly degenerated.

We recall that the two new fields associated with eigenvalues $\lambda_{2}, \lambda_{6}$ will inhibit the use of classical Eulertype solvers afterwards.

Property 4

The one-dimensional Riemann problem associated with the homogeneous system (13) projected along the direction aligned with $\mathbf{n}$ admits a unique solution with no vacuum occurence, provided that the initial data $\left(\mathbf{W}_{L}, \mathbf{R}_{L}, \mathbf{W}_{R}, \mathbf{R}_{R}\right)$ on each side of the initial discontinuity comply with the condition:

$$
\left(U_{n}\right)_{R}-\left(U_{n}\right)_{L}<\int_{0}^{\alpha_{L}}\left(\frac{c_{1}\left(s_{L}, \alpha\right)}{\alpha}\right) d(\alpha)+\int_{0}^{\alpha_{R}}\left(\frac{c_{1}\left(s_{R}, \alpha\right)}{\alpha}\right) d(\alpha)
$$

setting $s=R_{n n} /(\alpha)^{2}$. This result is valid for weak shocks only. The components of the kinetic tensor comply with the realisability concept. If the condition (26) is violated, a vacuum of particles occurs in the solution.

In practice, the solution of the one-dimensional Riemann problem associated with the projection of the $2 \mathrm{D}$ system consists in two uncoupled substeps. A straightforward consequence is that the resolution of the exact problem in the $2 \mathrm{D}$ framework is indeed feasable.

\section{Numerical schemes}

Following the work ${ }^{13}$, the system of equations (11) is treated within the framework of the methods for hyperbolic systems. For the sake of simplicity, we present the numerical scheme used in the case of 1-D computations. However, the generalisation to the multi-dimensional case is straightforward. The numerical scheme used to compute fluxes is an hybridization of the approximate Godunov scheme which has been introduced in ${ }^{14,15}$ with the exact Godunov solver. ${ }^{16}$ This simply means that the interface states occuring in the numerical fluxes are computed using either: (i) the exact solution of the one-dimensional Riemann problem when the condition (20) is violated, or: (ii) the approximate solution of the one-dimensional Riemann problem -described below- when the condition (20) holds. We emphasize that the transition between formulas is continuous. For $2 D$ computations, a similar procedure is applied, considering (26) instead of (20) of course. One main advantage is that it enables to cope with vacuum areas in an accurate way. The 
increase in CPU time is less than $5 \%$.

In one dimension, the homogeneous part of the set of PDEs can be written in a compact form as

$$
\begin{aligned}
& (\alpha)_{t}+(\alpha U)_{x}=0 \\
& (\alpha U)_{t}+\left(\alpha U^{2}+\alpha R\right)_{x}=0 \\
& (\alpha R)_{t}+(\alpha U R)_{x}+2 \alpha R U_{x}=0,
\end{aligned}
$$

where the subscripts represent the partial derivatives.

A change of variables is proposed, the system is rewritten with respect to the new variables:

$$
\mathbf{y}=(S, \Pi, U), \text { with } S=R \alpha^{-2}, \Pi=\alpha R .
$$

In this basis, the system has the matrix form $\mathbf{y}_{t}+\mathbf{A} \mathbf{y}_{x}=0$, with

$$
\mathbf{A}=\left(\begin{array}{ccc}
U & 0 & 0 \\
0 & U & 3 \Pi \\
0 & \alpha^{-1} & U
\end{array}\right)
$$

The eigenvalues are

$$
\left\{\begin{array}{l}
\lambda_{1}=U-c \\
\lambda_{2}=U \\
\lambda_{3}=U+c,
\end{array}\right.
$$

where $c^{2}=\frac{3 \Pi}{\alpha}$. The corresponding right eigenvectors are

$$
\left\{\begin{array}{l}
r_{1}=(0,-\alpha c, 1) \\
r_{2}=(1,0,0) \\
r_{3}=(0, \alpha c, 1),
\end{array}\right.
$$

The matrix of the eigenvectors and its inverse are

$$
\begin{gathered}
\boldsymbol{\Omega}=\left(\begin{array}{ccc}
0 & 1 & 0 \\
-\alpha c & 0 & \alpha c \\
1 & 0 & 1
\end{array}\right) \\
\boldsymbol{\Omega}^{-1}=\frac{1}{2 \alpha c}\left(\begin{array}{ccc}
0 & -1 & \alpha c \\
2 \alpha c & 0 & 0 \\
0 & 1 & \alpha c
\end{array}\right)
\end{gathered}
$$

At each time step, the following linearised Riemann problem is solved exactly

$$
\begin{cases}\mathbf{y}_{t} & +\mathbf{A}(\hat{\mathbf{y}}) \mathbf{y}_{x}=0 \\ \hat{\mathbf{y}} & =\frac{1}{2}\left(\mathbf{y}_{R}+\mathbf{y}_{L}\right)\end{cases}
$$

The solution is:

$$
\mathbf{y}(x / t)=\mathbf{y}_{L}+\sum_{\lambda_{k<x / t<\lambda_{k+1}}} b_{k} \hat{r}_{k} .
$$

The coefficients $b_{k}$ are computed by inverting the relation

$$
\mathbf{y}_{R}-\mathbf{y}_{L}=\sum_{k=1}^{3} b_{k} \hat{r}_{k}
$$

$$
6 \text { of } 11
$$


and, therefore, they are given by

$$
\left(\begin{array}{c}
b_{1} \\
b_{2} \\
b_{3}
\end{array}\right)=\boldsymbol{\Omega}^{-1}\left(\mathbf{y}_{R}-\mathbf{y}_{L}\right)=\frac{1}{2 \alpha c}\left(\begin{array}{c}
-[\Pi]_{L}^{R}+\alpha c[U]_{L}^{R} \\
2 \alpha c[S]_{L}^{R} \\
{[\Pi]_{L}^{R}+\alpha c[U]_{L}^{R}}
\end{array}\right)
$$

We now introduce notations:

$$
\mathbf{Z}^{\mathbf{t}}=(\alpha, \alpha \mathbf{U}, \boldsymbol{\Pi}) \quad \mathbf{g}(\mathbf{Z})^{\mathbf{t}}=\left(\alpha \mathbf{U}, \alpha \mathbf{U}^{2}+\boldsymbol{\Pi}, \mathbf{U} \boldsymbol{\Pi}\right)
$$

The numerical flux is defined as

$$
\mathbf{G}_{\mathbf{i}+\mathbf{1} / \mathbf{2}}=\mathrm{g}\left(\mathbf{Z}_{\mathbf{i}+\mathbf{1} / \mathbf{2}}^{*}\right)
$$

The approximate interface state $\mathbf{Z}_{\mathbf{i}+\mathbf{1} / \mathbf{2}}^{*}$ reads:

$$
\mathbf{Z}_{\mathbf{i}+\mathbf{1} / \mathbf{2}}^{*}=\mathbf{Z}^{\text {sol.ofRP }}\left(\left(\mathbf{x}-\mathbf{x}_{\mathbf{i}+\mathbf{1} / \mathbf{2}}\right) / \mathbf{t}=\mathbf{0}, \mathbf{Z}_{\mathbf{i}}, \mathbf{Z}_{\mathbf{i}+\mathbf{1}}\right)
$$

where $Z^{\text {sol.of } R P}$ denotes the exact solution of the linearized Riemann problem (36).

In compact and discrete form, the solution of this set of equations is advanced in time by the scheme

$$
\Delta x_{i}\left(\mathbf{Z}_{i}^{n+1}-\mathbf{Z}_{i}^{n}\right)+\Delta t^{n}\left(\mathbf{G}_{i+1 / 2}-\mathbf{G}_{i-1 / 2}\right)+\Delta t^{n} \int_{x_{i-1 / 2}}^{x_{i+1 / 2}}\left(\begin{array}{c}
0 \\
0 \\
2 \Pi U_{x}
\end{array}\right) d x=0
$$

where the numerical fluxes $\mathbf{G}$ at cell interfaces are computed by solving the Riemann problem as explained previously. The last integral is approximated following:

$$
\int_{x_{i-1 / 2}}^{x_{i+1 / 2}} 2 \Pi U_{x} d x=\left(\Pi_{i-1 / 2}^{*}+\Pi_{i+1 / 2}^{*}\right)\left(U_{i+1 / 2}^{*}-U_{i-1 / 2}^{*}\right)
$$

Then, the non-homogeneous terms of the system (11) are treated as source terms in a standard implicit way.

\section{Results}

In this section, we present an analysis of the stability of our algorithm for a given hybrid model. This analysis has a three-fold objective:

1. to check the stability of the numerical scheme when the kinetic tensor is noisy: the introduction of a noise in the system should not affect the non linear stability of the discrete approximation;

2. to verify that results given by the numerical scheme depend continuously on the level of noise.

3. to assess the ability of the numerical approach to smooth the noise, regardless of the mesh size, at least outside the regions where the noise is put in.

In that sense, we have chosen rather severe test-cases where the noise is introduced at each time-step with the same amplitude, while refining the mesh. Moreover, we wish to evaluate the physical error due to the noise, where the physical error is defined as the difference between the actual solution and the solution with zero noise (considered as the exact one). Two one-dimensional test-cases are considered herein.

\section{A. Case 1}

In the first test case, constant initial conditions are taken: $\left\langle U_{x}\right\rangle=1, \alpha=10^{-2}$; the Reynolds stress provided by the Lagrangian solver is composed by a constant part $R_{11}=10^{-4}$ and a random forcing over-imposed. In the case of zero forcing, the initial conditions represent also the analytical solution for this simple test-case.

In figure 1, the initial Reynolds stress is shown for the two cases treated, with or without noise. The level of noise has been chosen to be of the order of $1 \%$, and it is only imposed within the region corresponding to $[0.25 ; 0.75]$. The corresponding results for the density are shown in figure 2 . The numerical scheme is stable, the physical error introduced by the noise is reduced during the time integration and the density presents a difference with respect to the analytical solution of only $2 * 10^{-2 \%}$. 


\section{B. Case 2}

The second test-case is physically significant and is severe for testing the capacity of our numerical method of absorbing the oscillations created by the noise. The test-case represents a classical shock-tube, the velocity is initially put to zero everywhere. The initial discontinuity is placed in the middle of the domain; the left state is defined by :

$$
\alpha_{L}=5 * 10^{-2}, R_{11, L}=\alpha_{L} * 10^{-2}, U_{L}=0
$$

the right state is defined by :

$$
\alpha_{R}=1 * 10^{-2}, R_{11, R}=\alpha_{R} * 10^{-2}, U_{R}=0 .
$$

In figure 3, the temporal evolution of the solution for a Reynolds stress perturbed by a noise of $1 \%$ is shown. The noise is strongly diminished on the right side of the initial discontinuity, while a greater error is found in the left side. It looks as if the right-going shock wave is "cleaning" the noisy region. On the left side of the initial discontinuity, it clearly appears that the noise polluting the 1-rarefaction wave is smoothed by the whole scheme, and this is particularly obvious when the wave has left the noisy domain $(x<0.25)$. Globally speaking, the numerical scheme is found to be completely stable and accurate with respect to the physical error. In the figure 4 , the solution without noise and the solution with a noise of $0.1 \%, 1 \%$ and $5 \%$ are shown at the time $t=250$. The simulation with a noise of $0.1 \%$ is almost not distinguishable from the exact solution. The simulation with a noise of $1 \%$ shows some differences in the left side, but it remains in good agreement. The results with a noise of $5 \%$ show a stable solution but affected by an important error. In figure 5, the same configuration is shown for the mean particle velocity. The solution is always stable. The accuracy remains satisfying until the level of noise is bounded to be a few percent.

\section{Conclusion}

A complete new hybrid method for two-phase flows has been proposed, together with a new algorithm. The latter has been evaluated with special focus on its ability to cope with a noisy kinetic tensor. The solver is indeed stable with respect to noisy data, and the numerical approximations depend continuously on the level of noise introduced. These two basic features confirm that the approach is worth pursuing. Moreover, the numerical scheme has shown a good capacity to smooth noisy solutions, even when very refined meshes are used in order to make discretization errors negligible. The approach also holds in a multi-dimensional framework.

\section{Acknowledgments}

The first and second authors receive financial support through the NEPTUNE project and the MOFPHET$\mathrm{C}$ project respectively. The third author was funded by a Marie-Curie EU fellowship. The NEPTUNE project is financially supported by CEA (Commissariat à l'Energie Atomique), EDF (Electricité de France), IRSN (Institut de Radioprotection et Sûreté Nucléaire) and AREVA-NP.

\section{References}

\footnotetext{
${ }^{1}$ M. Muradoglu, P. Jenny, S. B. Pope, and D. A. Caughey. A consistent hybrid finite volume/particle method for the pdf equations of turbulent reactive flows. J. Comput. Phys., 154:342-371, 1999.

${ }^{2}$ J.M. Herard. A numerical tool to compute hybrid euler lagrange compressible models. In AIAA paper 2006-2872, San Fransisco, California, June 5-8 2006. 36th AIAA Fluid Dynamics Conference, http//www.aiaa.org.

${ }^{3}$ J.L. Lumley. Computational modelling of turbulent flows. Advances in Applied Mechanics, 18:123-176, 1978.

${ }^{4}$ J-P. Minier and E. Peirano. The pdf approach to polydispersed turbulent two-phase flows. Physics Reports, 352(1-3):1$214,2001$.

${ }^{5}$ E. Peirano, S. Chibbaro, J. Pozorski, and J.-P. Minier. Mean-field/pdf numerical approach for polydispersed turbulent two-phase flows. Progress Energy Combustion Science, 32:315-371, 2006.

${ }^{6}$ S. Chibbaro and J.P. Minier. Analysis of hybrid moments/pdf approach to turbulent two-phase flows in the tracer limit. Technical report, MFEE, Electricité de France, Chatou, France, 2006.
} 


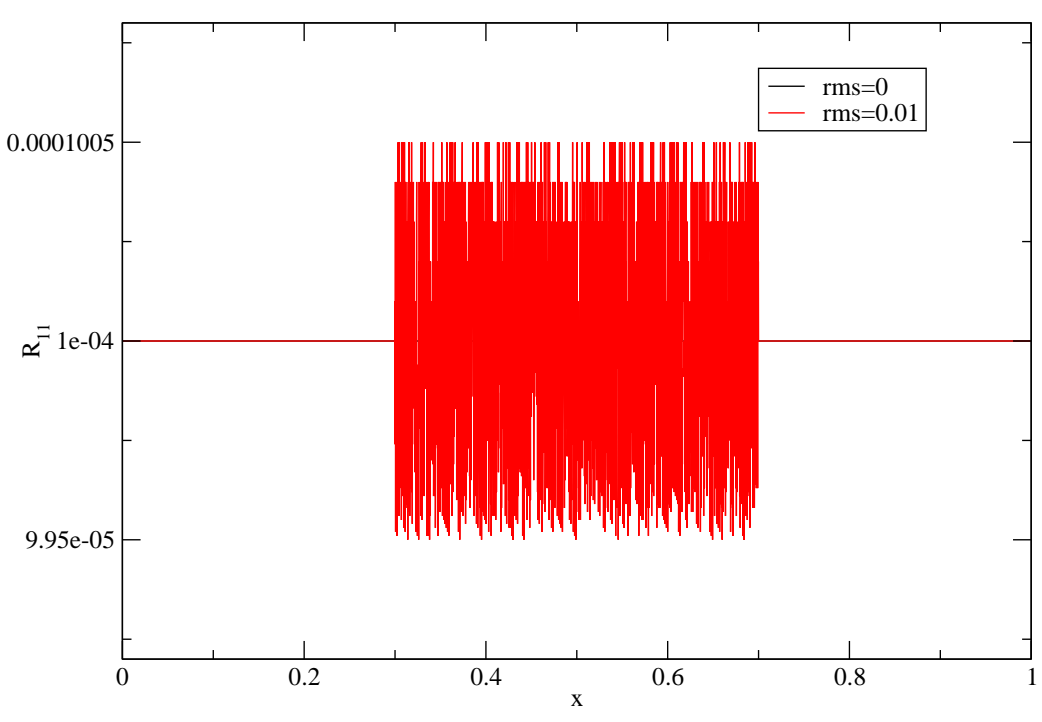

Figure 1. Initial conditions for the kinetic tensor, in the first test-case.

${ }^{7}$ S. Chibbaro, J.-P. Minier, and J.M. Hérard. Consistency and mass continuity issues in hybrid pdf simulations of turbulent two-phase flows. Technical report, MFEE, Electricité de France, Chatou, France, 2006.

${ }^{8}$ G. Brun, J.M. Hérard, D. Jeandel, and M. Uhlmann. An approximate roe type riemann solver for a class of realisable second order closures. Int. J. Comp. Fluid Dyn., 13(3):223-249, 2000.

${ }^{9} \mathrm{~B}$. Audebert and F. Coquel. Travelling wave analysis for a class of reynolds stress models. in preparation, 2007.

${ }^{10} \mathrm{~B}$. Audebert. Contribution à l'analyse des modèles aux tensions de Reynolds pour l'interaction choc turbulence. PhD thesis, Université Pierre et Marie Curie, Paris, France, December 19, 2006.

${ }^{11}$ E. Peirano and J.-P. Minier. Probabilistic formalism and hierarchy of models for polydispersed turbulent two-phase flows. Phys. Rev. E, 65:046301, 2002.

${ }^{12}$ J.M. Hérard. Modèles au second ordre réalisables non dégénérés pour les écoulements turbulents incompressibles. CRAS Paris, IIb(322):371-377, 1996.

${ }^{13}$ C. Berthon, F. Coquel, J.M. Hérard, and M. Uhlmann. An approximate solution of the riemann problem for a realisable second-moment turbulent closure. Shock waves, 11(4):245-269, 2002.

${ }^{14} \mathrm{~T}$. Buffard, T. Gallouet, and J.M. Hérard. A sequel to a rough godunov scheme. application to real gases. Computers and Fluids, 29(7):813-847, 2000.

${ }^{15}$ T. Gallouet, J.M. Hérard, and N. Seguin. On the use of symetrizing variables for vacuum. Calcolo, 40(3):163-194, 2003.

${ }^{16} \mathrm{~S}$. Godunov. A difference method for numerical calculation of discontinuous equations of hydrodynamics. Mat. Sb., in Russian:271-300, 1959. 


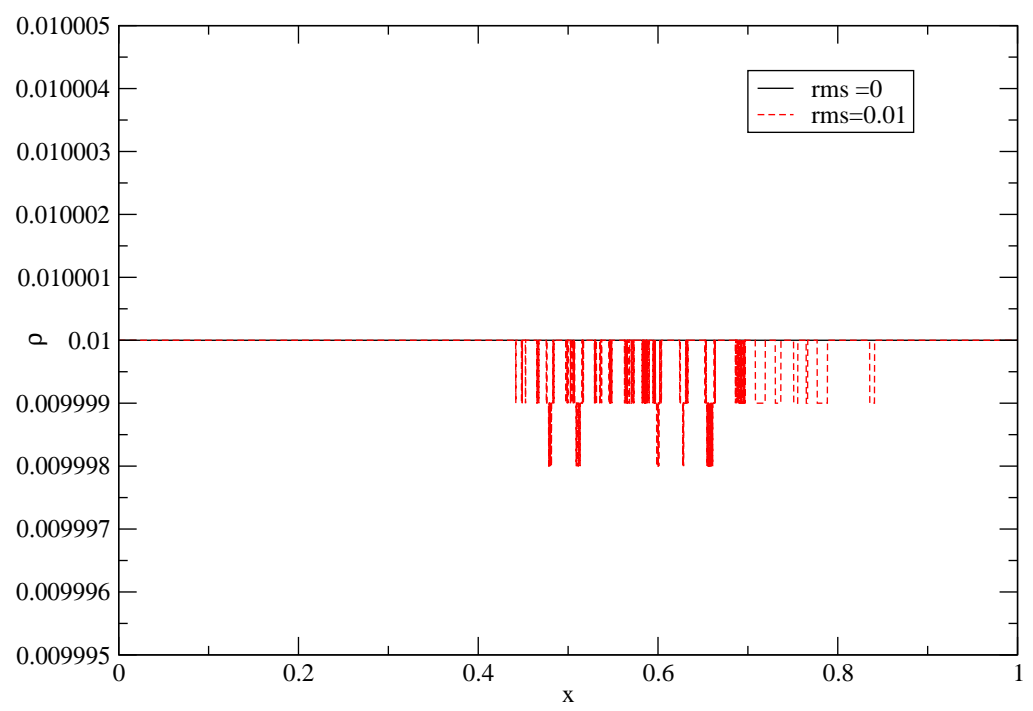

Figure 2. Density solution, in the first test-case.

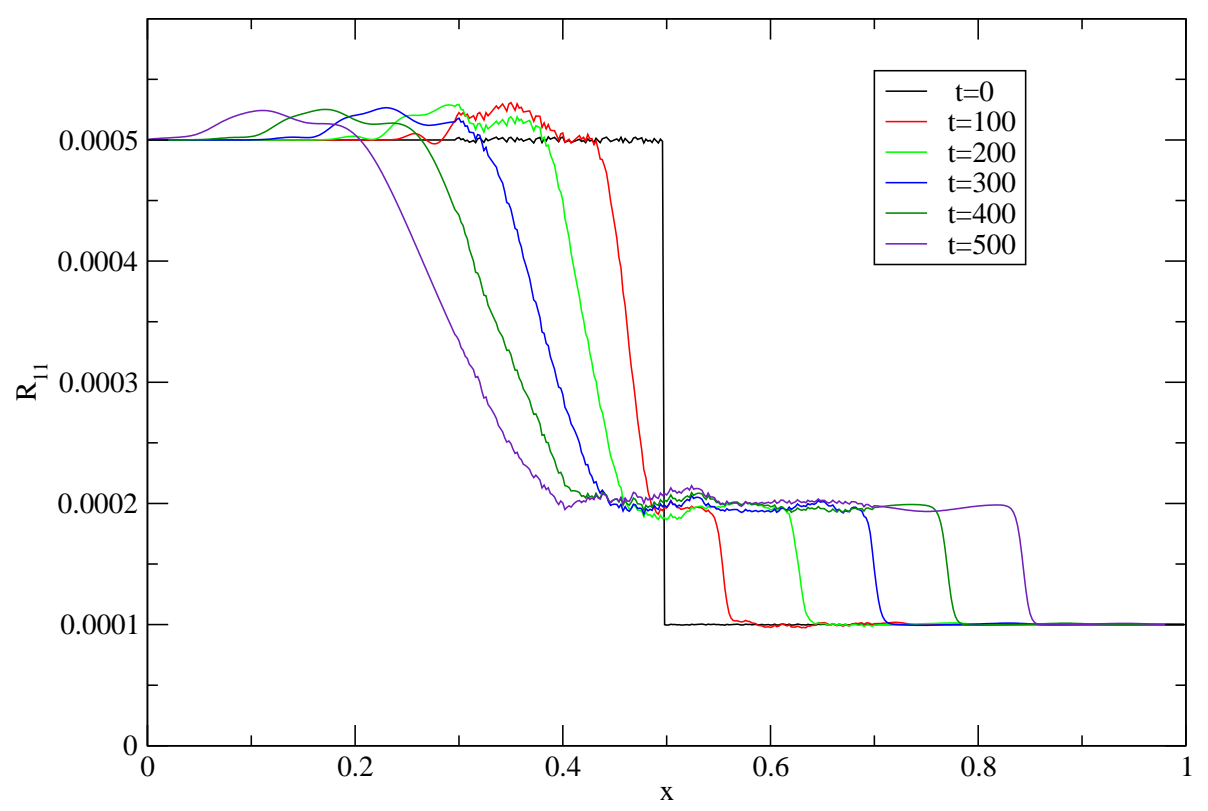

Figure 3. Temporal evolution of the kinetic tensor in a shock tube.

10 of 11 
$\mathrm{t}=250$

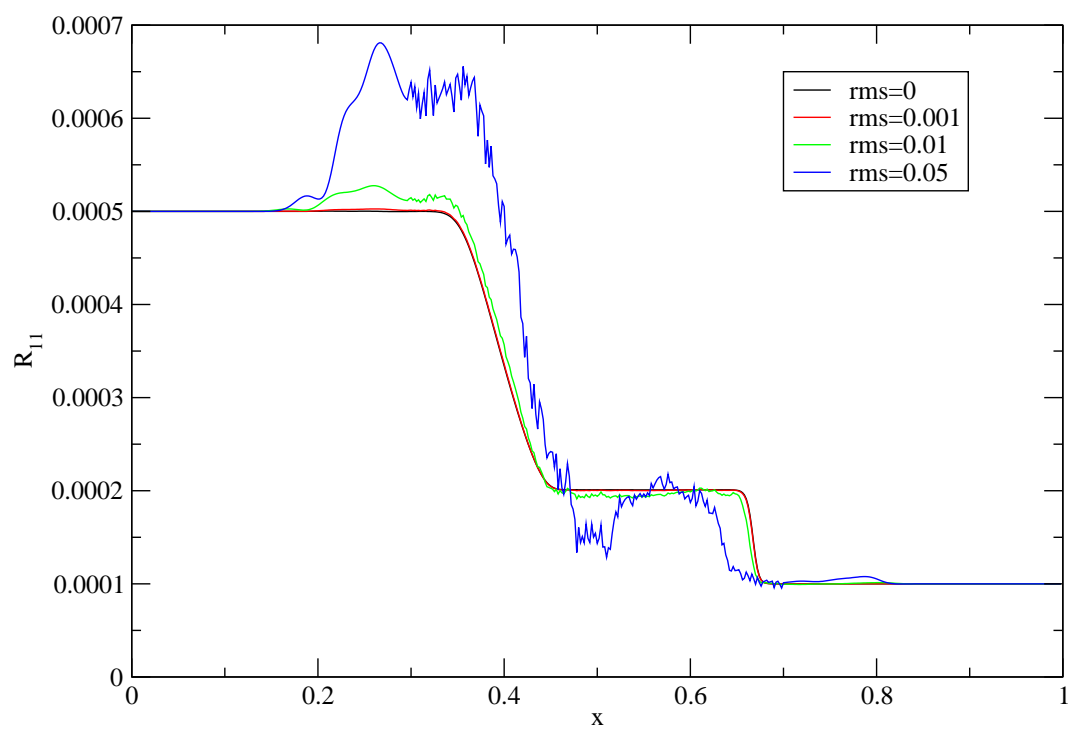

Figure 4. The Kinetic tensor $t=250$ for different levels of noise.

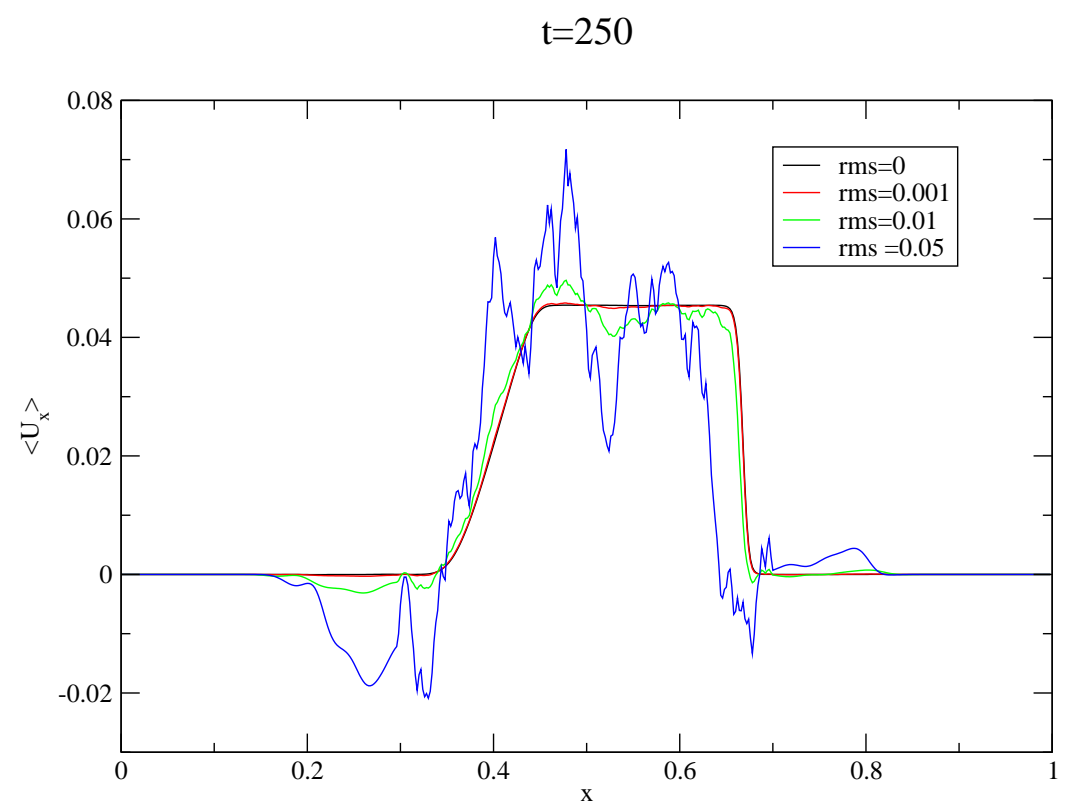

Figure 5. Particle mean velocity at time $t=250$ for different levels of noise.

11 of 11 